\title{
Turbulent Statistics from Time-Resolved PIV Measurements of a Jet Using Empirical Mode Decomposition
}

\author{
Milo D. Dahl \\ NASA Glenn Research Center, Cleveland, OH, 44135, USA
}

Empirical mode decomposition is an adaptive signal processing method that when applied to a broadband signal, such as that generated by turbulence, acts as a set of band-pass filters. This process was applied to data from time-resolved, particle image velocimetry measurements of subsonic jets prior to computing the second-order, two-point, space-time correlations from which turbulent phase velocities and length and time scales could be determined. The application of this method to large sets of simultaneous time histories is new. In this initial study, the results are relevant to acoustic analogy source models for jet noise prediction. The high frequency portion of the results could provide the turbulent values for subgrid scale models for noise that is missed in large-eddy simulations. The results are also used to infer that the cross-correlations between different components of the decomposed signals at two points in space, neglected in this initial study, are important. 


\title{
Turbulent Statistics from Time-Resolved PIV Measurements of a Jet Using Empirical Mode Decomposition
}

\author{
Milo D. Dahl
}

NASA Glenn Research Center

18th AIAA/CEAS Aeroacoustics Conference June 4-6, 2012

Supported by NASA Fundamental Aeronautics/Supersonics Project 


\section{Introduction}

- Provide statistical properties of turbulence to aid in modeling jet noise sources

- Acoustic analogy methods and subgrid models

- Two-point, space-time correlations

- numerically - direct numerical simulation

- experimentally - time-resolved PIV

- Method-empirical mode decomposition

- Automatic filtering for small scales - subgrid models

- Presentation:

(1) Time-resolved PIV measurements

(2) Empirical mode decomposition - new application

(3) Initial analysis of and results from correlation calculations

(4) Comments on method and correlations 


\section{Overview of Time-Resolved PIV}

High sampling rate means small field of view

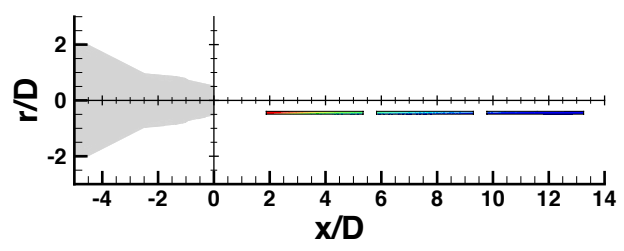

$25 \mathrm{kHz}$

$\sim 1 \mathrm{sec}, 24993$ points

$178.85 \mathrm{~mm}$ by $5.18 \mathrm{~mm}$

70 by 5 points

$\Delta \mathrm{x} / \mathrm{D}=0.0510$

$\Delta \mathrm{r} / \mathrm{D}=0.0255$

Flow conditions for converging nozzle $D=50.8 \mathrm{~mm}$

\begin{tabular}{|c|c|c|c|c|c|c|c|}
\hline Case & $T_{t} / T_{\infty}$ & $T_{s} / T_{\infty}$ & $P_{t} / P_{\infty}$ & $\mathrm{M}_{J}$ & $U_{J} / c_{\infty}$ & $U_{J}(\mathrm{~m} / \mathrm{s})$ & $f U_{J} / D$ \\
\hline SP3 & 1.00 & 0.95 & 1.20 & 0.51 & 0.50 & 172.8 & 3.67 \\
SP7 & 1.00 & 0.84 & 1.85 & 0.98 & 0.90 & 310.0 & 2.05 \\
\hline
\end{tabular}




\section{Mean Flow}

\section{SP7 jet with $M_{J}=0.98$}

Axial velocity contours

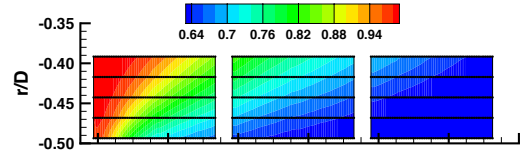

Axial velocity

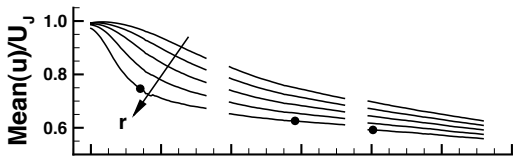

Turbulence intensities

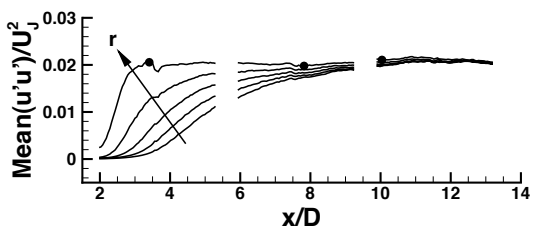

Reference points: $r / D=-0.49$

$$
x / D=3.41,7.82,10.0
$$




\section{Empirical Mode Decomposition (EMD)}

- Adaptive signal processing method for a general, non-stationary and nonlinear signal

- Huang, et al.: Proc. Roy. Society London, 1998

- Separates signal into basis functions (a posteriori, based on the signal, not a priori, based on harmonics)

- Defined by an algorithm, limited mathematical foundation

- Applied in vibrations, geophysics, biology, cosmology, finance, others

- Application to large set of simultaneous time histories all having the same broadband nature and then computing correlations is new 


\section{EMD Algorithm I}

Start with the signal: the axial velocity fluctuation after subtracting the mean axial velocity

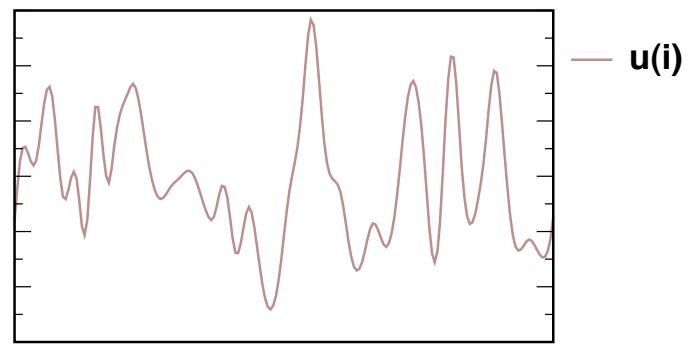




\section{EMD Algorithm I}

\section{Identify all maxima of the signal}

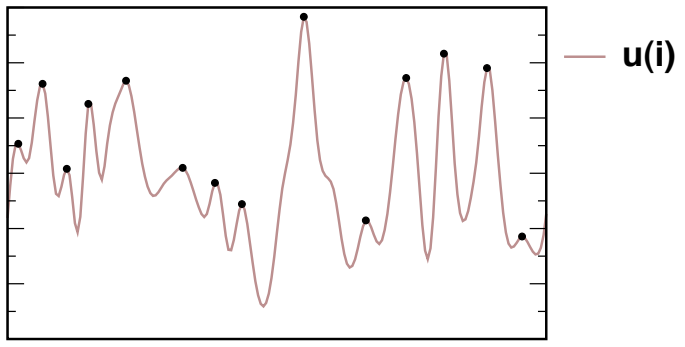




\section{EMD Algorithm I}

\section{Interpolating function found using cubic spline gives maximum of envelope}

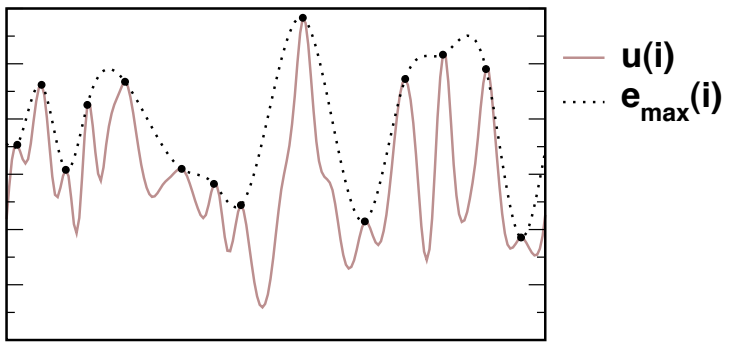




\section{EMD Algorithm I}

Repeat for the minimum envelope

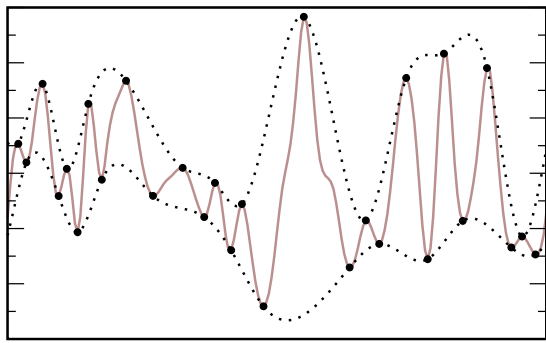

u(i)

$\mathbf{e}_{\text {max }}(\mathbf{i}), \mathbf{e}_{\text {min }}(\mathbf{i})$ 


\section{EMD Algorithm I}

Compute average of the envelope

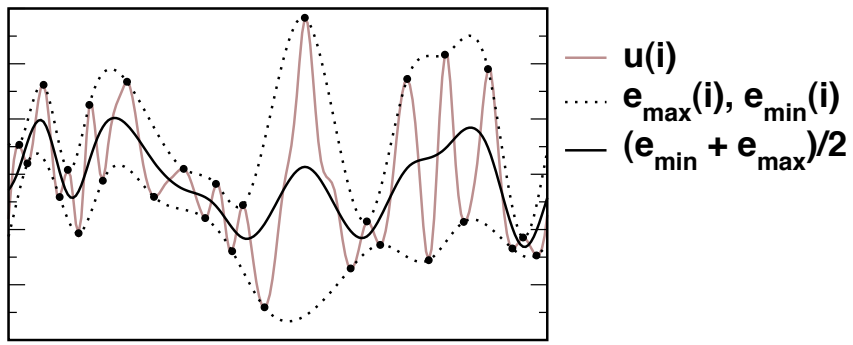




\section{EMD Algorithm II}

An interpretation of EMD is that it separates the local (high frequency) oscillations from the local (low frequency) trend

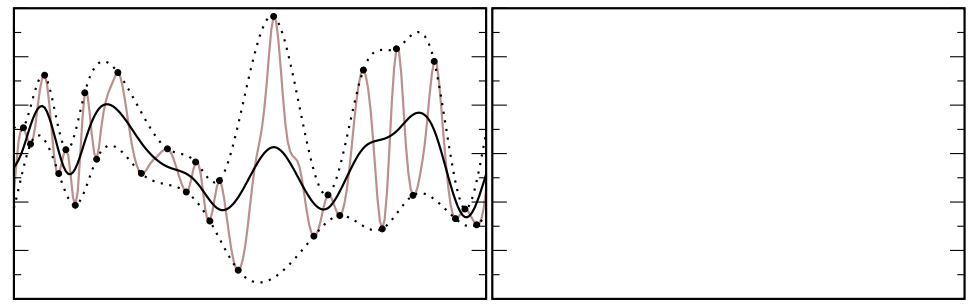




\section{EMD Algorithm II}

An interpretation of EMD is that it separates the local (high frequency) oscillations from the local (low frequency) trend

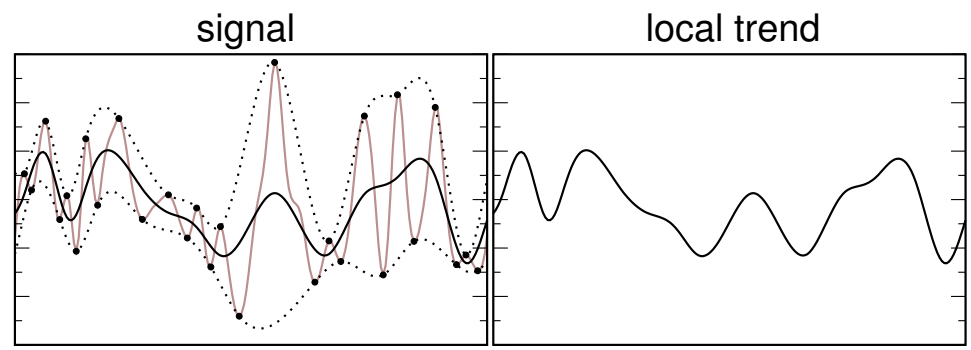




\section{EMD Algorithm II}

Subtract local trend from signal Ideally should get zero mean oscillation

Sift 1 local trend

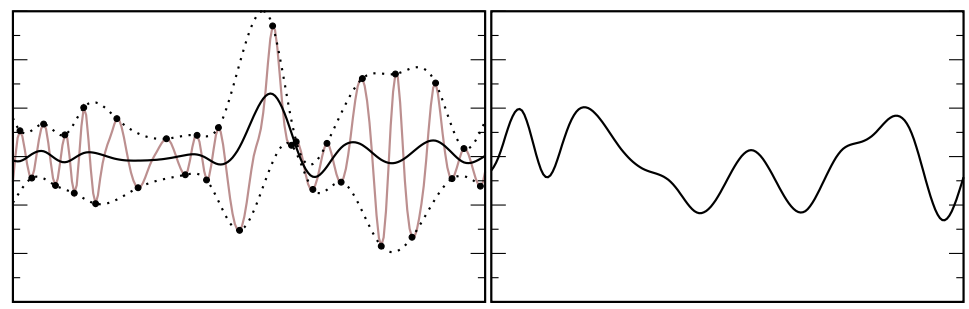




\section{EMD Algorithm II}

Subtract local trend from signal

Keep sifting

Sift 2

local trend

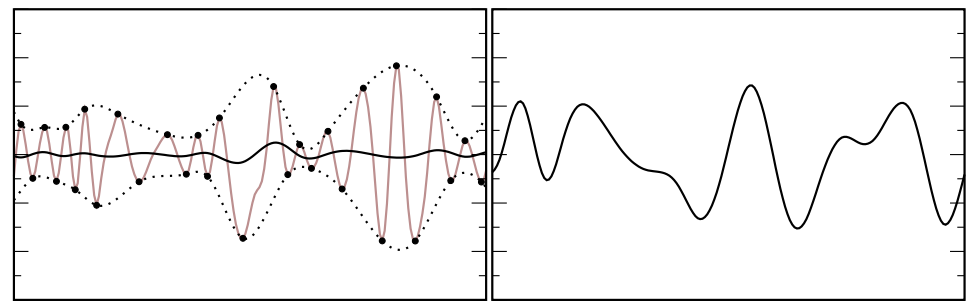




\section{EMD Algorithm II}

\section{Subtract local trend from signal Keep sifting}

Sift 3

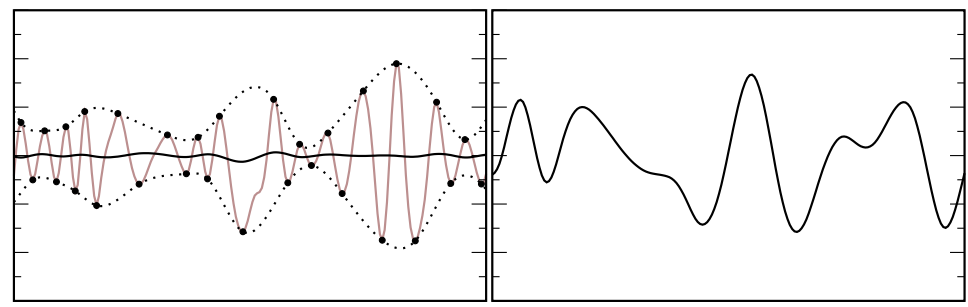




\section{EMD Algorithm II}

\section{Subtract local trend from signal Keep sifting}

Sift 4 local trend

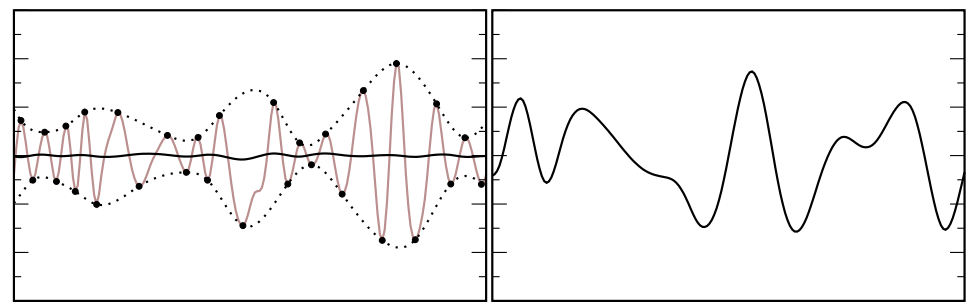




\section{EMD Algorithm II}

\section{Subtract local trend from signal Keep sifting}

Sift 5 local trend

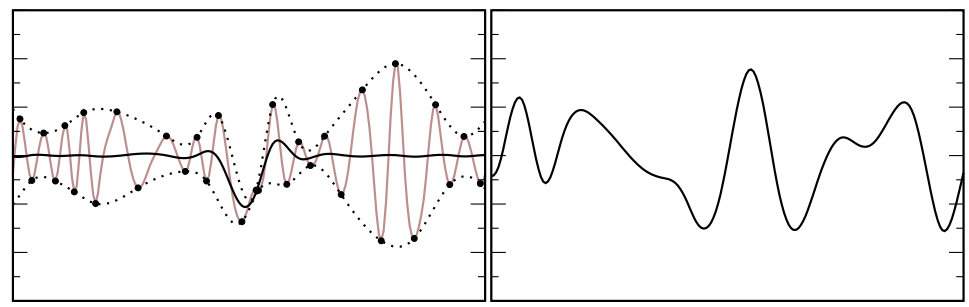




\section{EMD Algorithm II}

\section{Subtract local trend from signal Keep sifting}

Sift 6 local trend

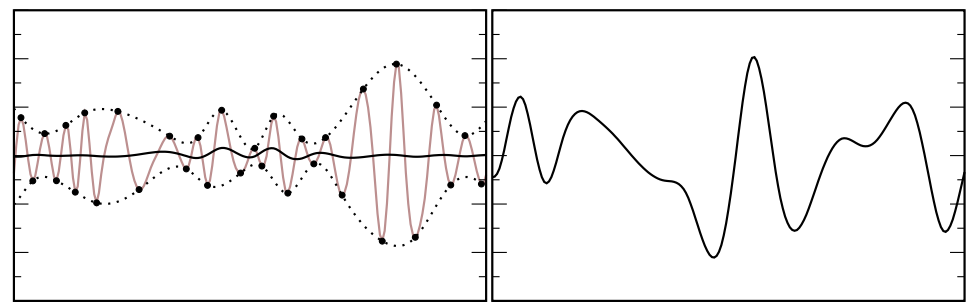




\section{EMD Algorithm II}

\section{Subtract local trend from signal Keep sifting}

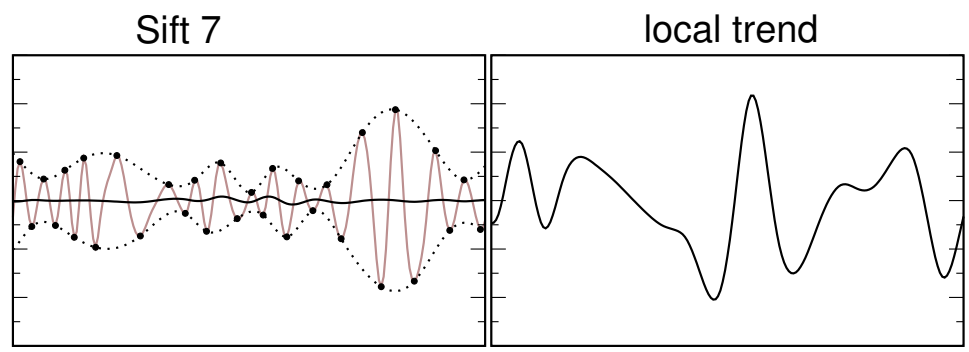




\section{EMD Algorithm II}

\section{Subtract local trend from signal Keep sifting}

Sift 8 local trend

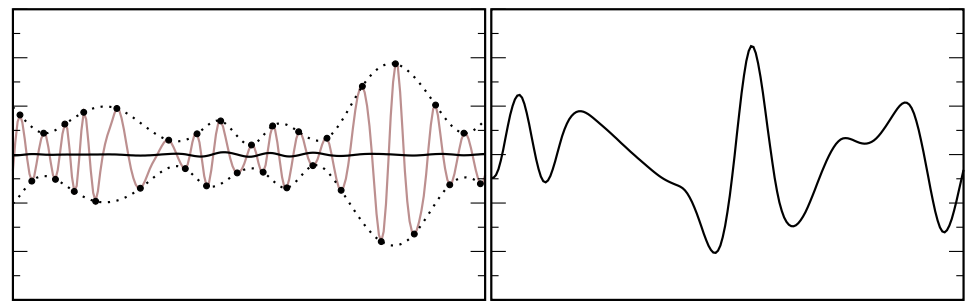




\section{EMD Algorithm II}

Subtract local trend from signal

Keep sifting

Sift 9

local trend

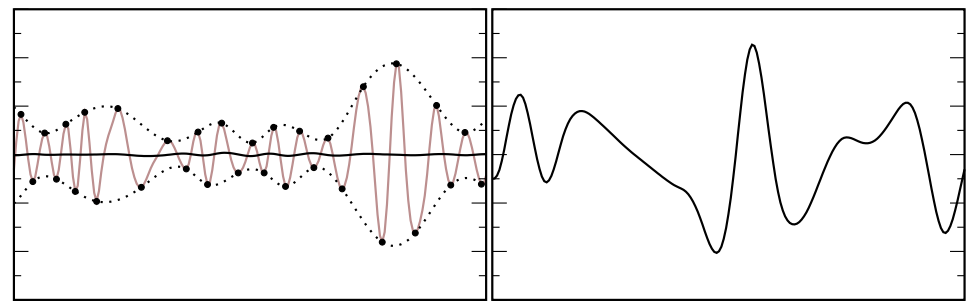




\section{EMD Algorithm II}

Subtract local trend from signal

Stop sifting

Sift 10

local trend

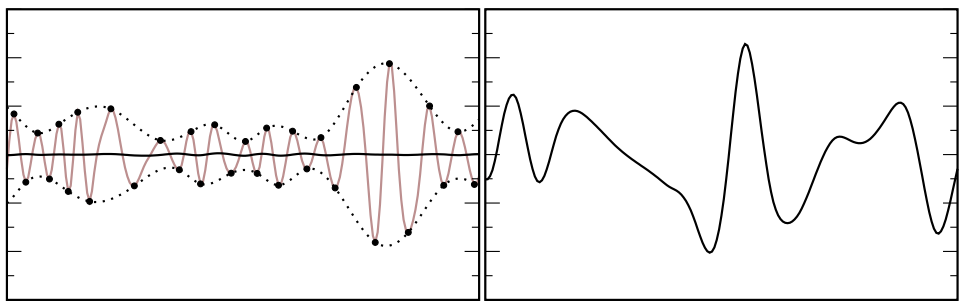

Intrinsic Mode Function (IMF) Process with EMD

- $\mid$ (extrema) - (zeroes) $\mid \leq 1 \quad$ to get next IMF

- Mean about zero

- Symmetric 


\section{Example Intrinsic Mode Functions (IMF)}

0.06 second time sample of a 1 second TR-PIV time history

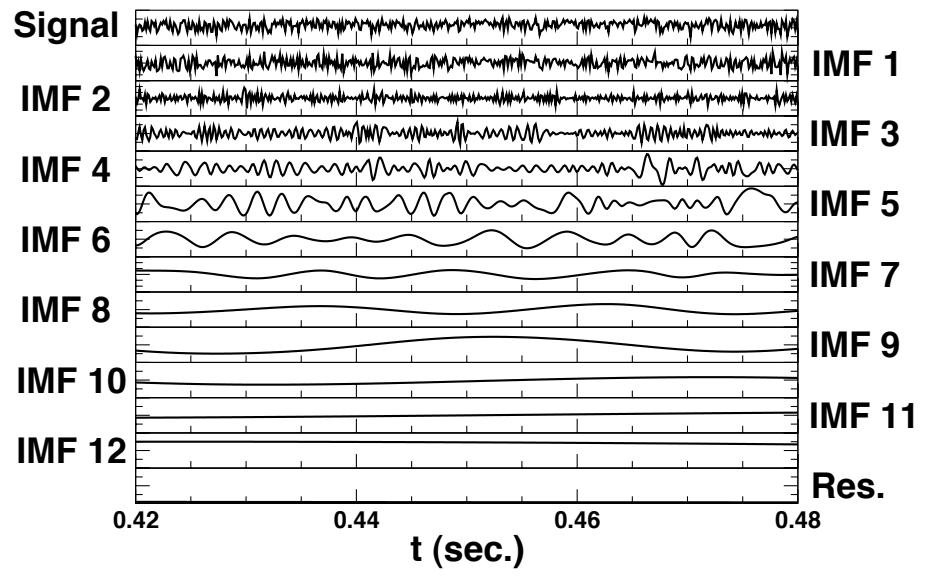

IMFs nearly orthogonal and uncorrelated: $u(t)=\sum_{n=1}^{N} C_{n}(t)+z_{N}(t)$ 


\section{Broadband Signal Processed by EMD}

EMD acts as a dyadic filter bank

- Set of overlapping band-pass filters

- half or double range of neighbor

- approx. constant shape on log scale

- mean frequencies $f_{n}^{c} \approx f_{o} 2^{-n}$ for $n$-th IMF

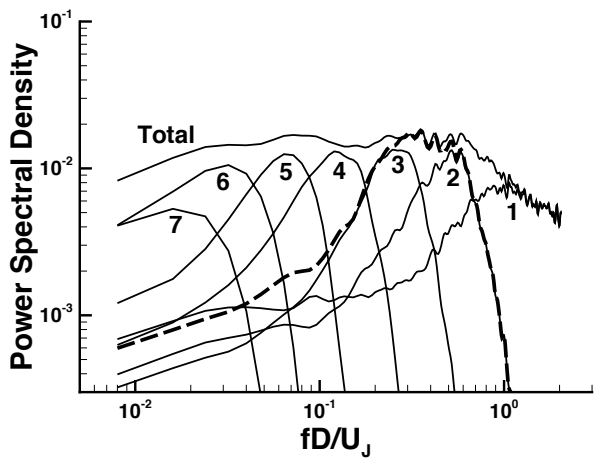

Dashed line: Add IMFs 2 and 3

Ref. Point: $x / D=3.41$ shear layer 


\section{Broadband Signal Processed by EMD}

EMD acts as a dyadic filter bank

- Set of overlapping band-pass filters

- half or double range of neighbor

- approx. constant shape on log scale

- mean frequencies $f_{n}^{c} \approx f_{o} 2^{-n}$ for $n$-th IMF

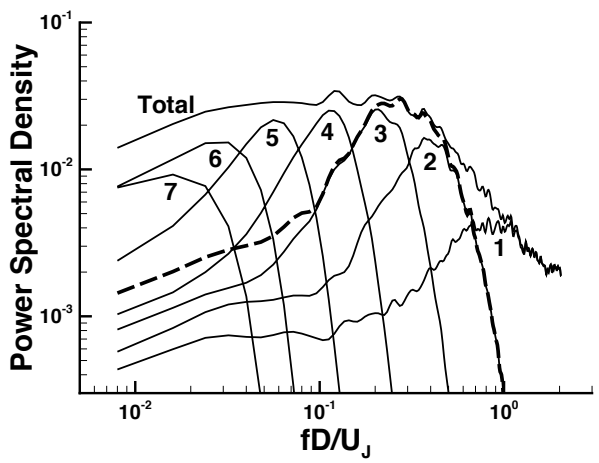

Dashed line:

Add IMFs 2 and 3

Ref. Point: $x / D=7.82$ end of potential core 


\section{Broadband Signal Processed by EMD}

EMD acts as a dyadic filter bank

- Set of overlapping band-pass filters

- half or double range of neighbor

- approx. constant shape on log scale

- mean frequencies $f_{n}^{c} \approx f_{o} 2^{-n}$ for $n$-th IMF

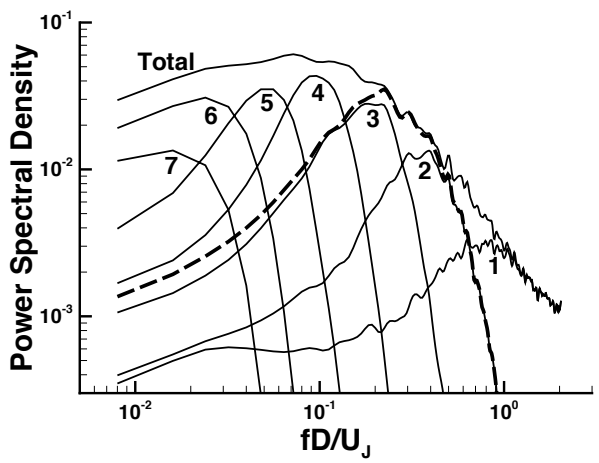

Dashed line:

Add IMFs 2 and 3

Ref. Point: $x / D=10.0$ downstream 


\section{Correlation I}

$$
\text { Total } \quad r_{11}\left(\boldsymbol{x}, \eta_{1}, \tau\right)=\frac{\overline{\overline{u_{1}^{\prime}(\boldsymbol{x}, t) \cdot u_{1}^{\prime}\left(\boldsymbol{x}+\eta_{1}, t+\tau\right)}}}{\sqrt{\overline{u_{1}^{\prime}(\boldsymbol{x}, t)^{2}} \cdot \overline{u_{1}^{\prime}\left(\boldsymbol{x}+\eta_{1}, t+\tau\right)^{2}}}}
$$

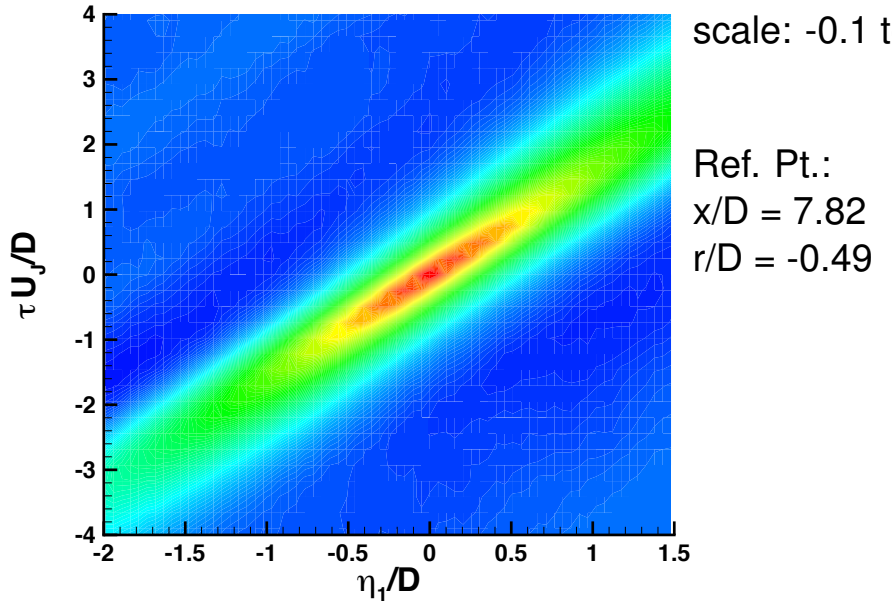




\section{Correlation I}

$$
\operatorname{IMF} 1 \quad r_{11 n}\left(\boldsymbol{x}, \eta_{1}, \tau\right)=\frac{\overline{C_{1 n}(\boldsymbol{x}, t) \cdot C_{1 n}\left(\boldsymbol{x}+\eta_{1}, t+\tau\right)}}{\sqrt{\overline{u_{1}^{\prime}(\boldsymbol{x}, t)^{2}} \cdot \overline{u_{1}^{\prime}\left(\boldsymbol{x}+\eta_{1}, t+\tau\right)^{2}}}}, n=1
$$

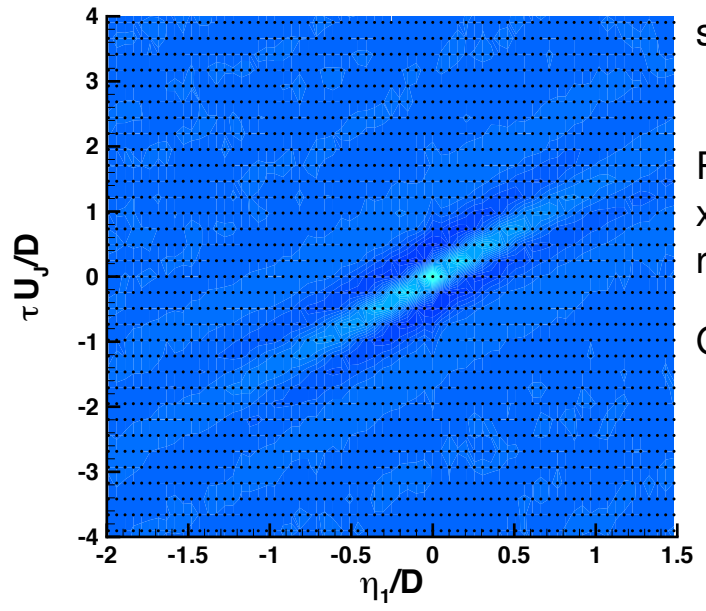

scale: -0.1 to 1

Ref. Pt.:

$\mathrm{x} / \mathrm{D}=7.82$

$r / D=-0.49$

Grid too coarse? 


\section{Correlation I}

IMF $2 \quad r_{11 n}\left(\boldsymbol{x}, \eta_{1}, \tau\right)=\frac{\overline{C_{1 n}(\boldsymbol{x}, t) \cdot C_{1 n}\left(\boldsymbol{x}+\eta_{1}, t+\tau\right)}}{\sqrt{\overline{u_{1}^{\prime}(\boldsymbol{x}, t)^{2}} \cdot \overline{u_{1}^{\prime}\left(\boldsymbol{x}+\eta_{1}, t+\tau\right)^{2}}}}, n=2$

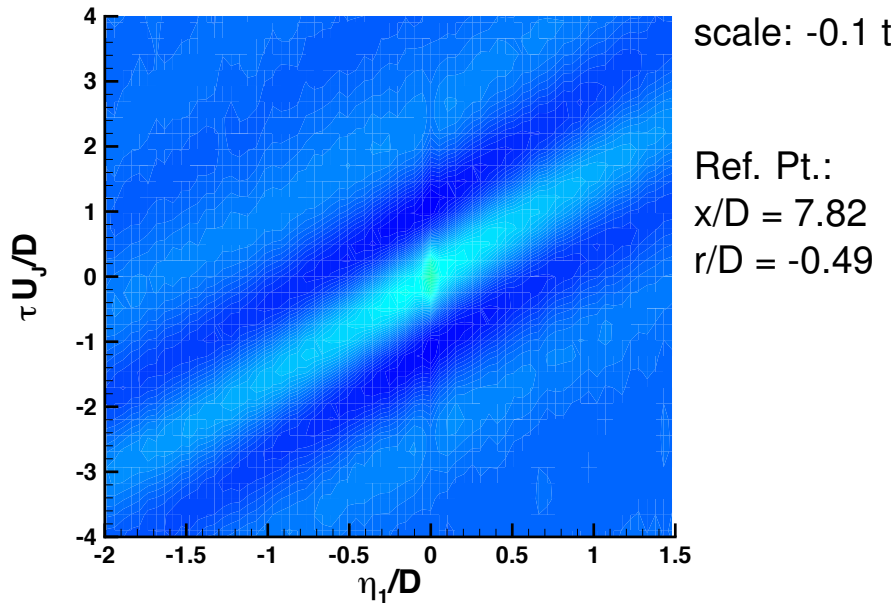




\section{Correlation I}

$\operatorname{IMF} 3 \quad r_{11 n}\left(\boldsymbol{x}, \eta_{1}, \tau\right)=\frac{\overline{C_{1 n}(\boldsymbol{x}, t) \cdot C_{1 n}\left(\boldsymbol{x}+\eta_{1}, t+\tau\right)}}{\sqrt{\overline{u_{1}^{\prime}(\boldsymbol{x}, t)^{2}} \cdot \overline{u_{1}^{\prime}\left(\boldsymbol{x}+\eta_{1}, t+\tau\right)^{2}}}}, n=3$

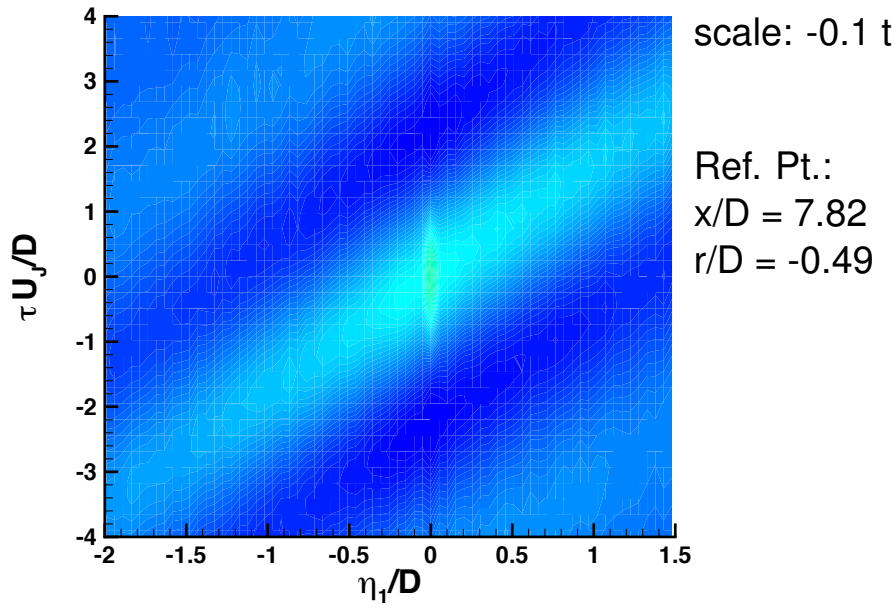




\section{Correlation I}

$$
\text { IMF } 4 \quad r_{11 n}\left(\boldsymbol{x}, \eta_{1}, \tau\right)=\frac{\overline{C_{1 n}(\boldsymbol{x}, t) \cdot C_{1 n}\left(\boldsymbol{x}+\eta_{1}, t+\tau\right)}}{\sqrt{\overline{u_{1}^{\prime}(\boldsymbol{x}, t)^{2}} \cdot \overline{u_{1}^{\prime}\left(\boldsymbol{x}+\eta_{1}, t+\tau\right)^{2}}}}, n=4
$$

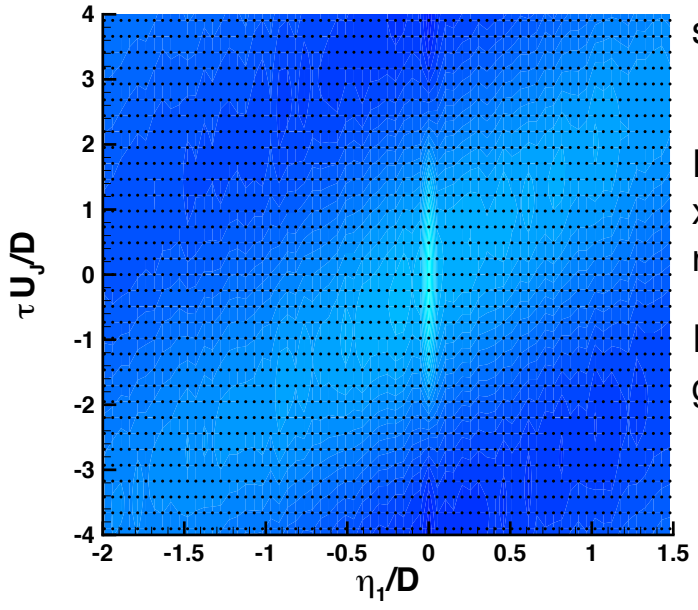

scale: -0.1 to 1

Ref. Pt.:

$x / D=7.82$

$r / D=-0.49$

Issue within 1 grid spacing 


\section{Integral Length and Time Scales}

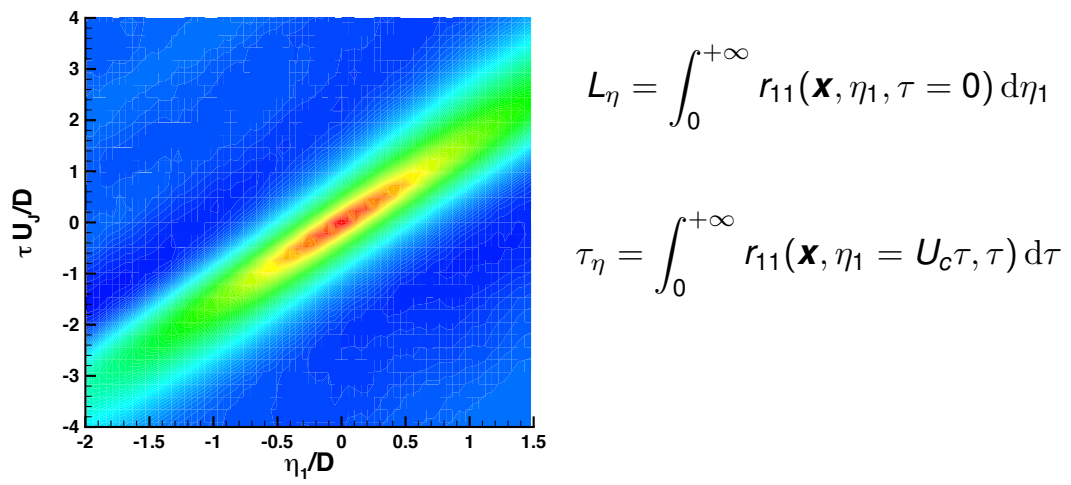

Estimate the integral length and time scales by determining how far it takes the correlation to decay by $1 / \mathrm{e}$ from the peak value. 


\section{Integral Length and Time Scales}

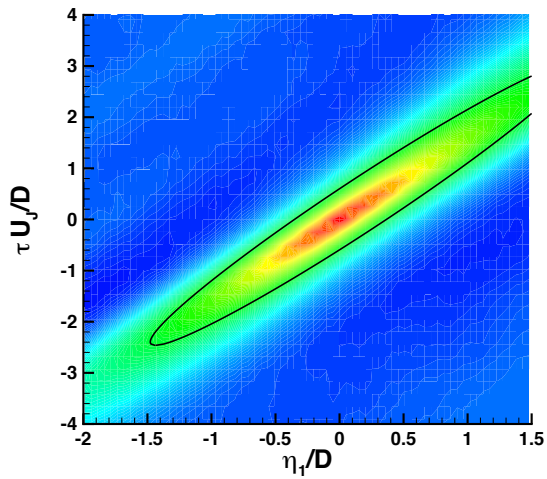

$$
\begin{gathered}
L_{\eta}=\int_{0}^{+\infty} r_{11}\left(\boldsymbol{x}, \eta_{1}, \tau=0\right) \mathrm{d} \eta_{1} \\
\tau_{\eta}=\int_{0}^{+\infty} r_{11}\left(\boldsymbol{x}, \eta_{1}=U_{c} \tau, \tau\right) \mathrm{d} \tau
\end{gathered}
$$

Estimate the integral length and time scales by determining how far it takes the correlation to decay by $1 / \mathrm{e}$ from the peak value. Contour resembles an ellipse. 


\section{Ellipse}

Fit an ellipse to the contour where the level is 1/e times the peak value of the correlation. Find coefficients $a$ to $f$.

$$
a\left(\frac{\eta_{1}}{D}\right)^{2}+b \frac{\eta_{1}}{D} \frac{\tau U_{J}}{D}+c\left(\frac{\tau U_{J}}{D}\right)^{2}+d \frac{\eta_{1}}{D}+e \frac{\tau U_{J}}{D}+f=0
$$

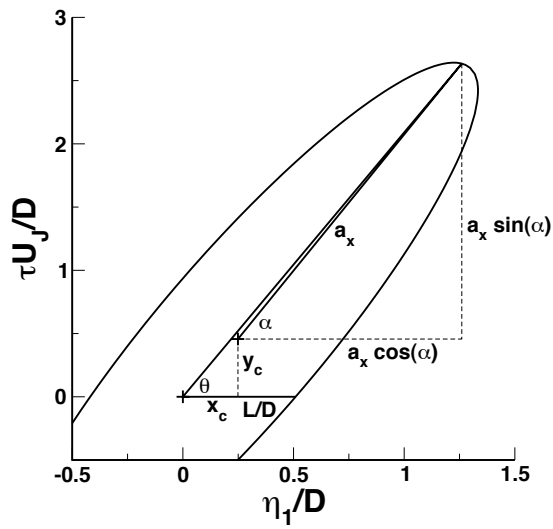

$$
\begin{gathered}
\frac{L}{D}=-\left(\frac{d}{2 a}\right)+\sqrt{\left(\frac{d}{2 a}\right)^{2}-\frac{f}{a}} \\
\frac{\tau U_{J}}{D}=a_{x} \sin \alpha+y_{c} \\
\frac{U_{c}}{U_{J}}=\frac{1}{\tan \theta}
\end{gathered}
$$




\section{Correlation II}

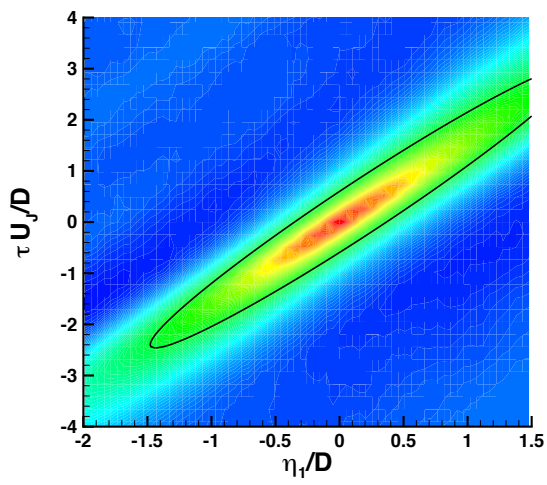

$$
\begin{array}{l|l} 
& \text { Total } \\
U_{c} / U_{J} & 0.596 \\
L_{\eta} / D & 0.366 \\
\tau_{\eta} U_{J} / D & 3.057
\end{array}
$$

Estimate:

$$
\begin{aligned}
& L_{\eta}=\int_{0}^{+\infty} r_{11}\left(\boldsymbol{x}, \eta_{1}, \tau=0\right) \mathrm{d} \eta_{1} \\
& \tau_{\eta}=\int_{0}^{+\infty} r_{11}\left(\boldsymbol{x}, \eta_{1}=U_{c} \tau, \tau\right) \mathrm{d} \tau \\
& \text { using ellipse eq. }
\end{aligned}
$$




\section{Correlation II}

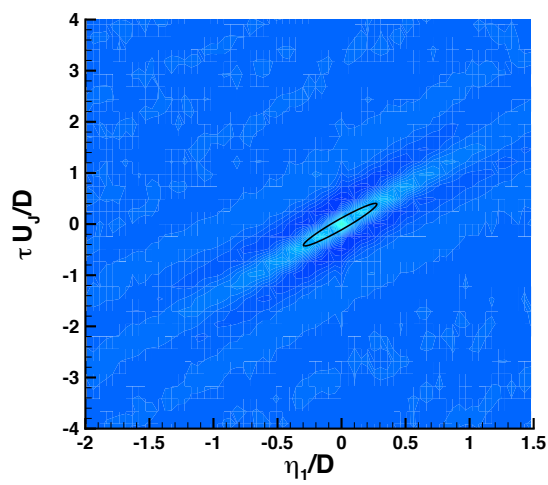

Estimate:

$$
\begin{aligned}
& L_{\eta}=\int_{0}^{+\infty} r_{11}\left(\boldsymbol{x}, \eta_{1}, \tau=0\right) \mathrm{d} \eta_{1} \\
& \tau_{\eta}=\int_{0}^{+\infty} r_{11}\left(\boldsymbol{x}, \eta_{1}=U_{c} \tau, \tau\right) \mathrm{d} \tau \\
& \text { using ellipse eq. }
\end{aligned}
$$

$$
\begin{array}{l|ll} 
& \text { Total } & \text { IMF 1 } \\
U_{c} / U_{J} & \mathbf{0 . 5 9 6} & \mathbf{0 . 6 7 0} \\
L_{\eta} / D & \mathbf{0 . 3 6 6} & \mathbf{0 . 0 7 6} \\
\tau_{\eta} U_{J} / D & \mathbf{3 . 0 5 7} & \mathbf{0 . 4 0 3}
\end{array}
$$




\section{Correlation II}

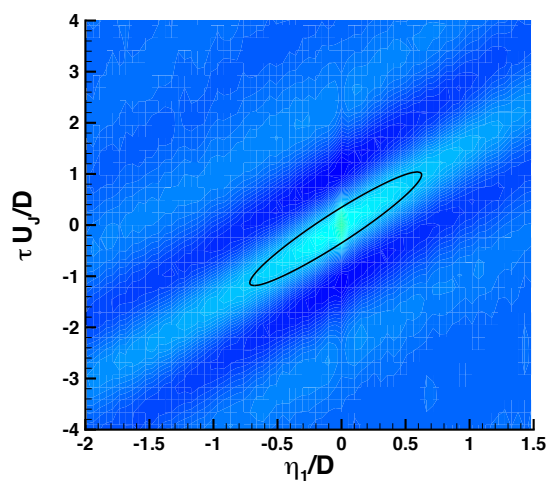

Estimate:

$$
\begin{aligned}
& L_{\eta}=\int_{0}^{+\infty} r_{11}\left(\boldsymbol{x}, \eta_{1}, \tau=0\right) \mathrm{d} \eta_{1} \\
& \tau_{\eta}=\int_{0}^{+\infty} r_{11}\left(\boldsymbol{x}, \eta_{1}=U_{c} \tau, \tau\right) \mathrm{d} \tau \\
& \text { using ellipse eq. }
\end{aligned}
$$

\begin{tabular}{l|lll} 
& Total & IMF 1 & IMF 2 \\
$U_{c} / U_{J}$ & $\mathbf{0 . 5 9 6}$ & $\mathbf{0 . 6 7 0}$ & $\mathbf{0 . 5 8 6}$ \\
$L_{\eta} / D$ & $\mathbf{0 . 3 6 6}$ & $\mathbf{0 . 0 7 6}$ & $\mathbf{0 . 2 0 6}$ \\
$\tau_{\eta} U_{J} / D$ & $\mathbf{3 . 0 5 7}$ & $\mathbf{0 . 4 0 3}$ & $\mathbf{1 . 0 3 6}$
\end{tabular}




\section{Correlation II}

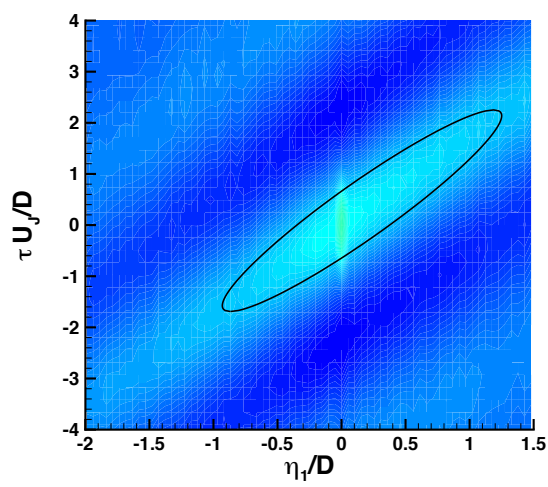

Estimate:

$$
\begin{aligned}
& L_{\eta}=\int_{0}^{+\infty} r_{11}\left(\boldsymbol{x}, \eta_{1}, \tau=0\right) \mathrm{d} \eta_{1} \\
& \tau_{\eta}=\int_{0}^{+\infty} r_{11}\left(\boldsymbol{x}, \eta_{1}=U_{c} \tau, \tau\right) \mathrm{d} \tau \\
& \text { using ellipse eq. }
\end{aligned}
$$

\begin{tabular}{l|llll} 
& Total & IMF 1 & IMF 2 & IMF 3 \\
$U_{c} / U_{J}$ & 0.596 & 0.670 & 0.586 & 0.540 \\
$L_{\eta} / D$ & 0.366 & 0.076 & 0.206 & 0.375 \\
$\tau_{\eta} U_{J} / D$ & 3.057 & 0.403 & 1.036 & 2.246
\end{tabular}




\section{Correlation II}

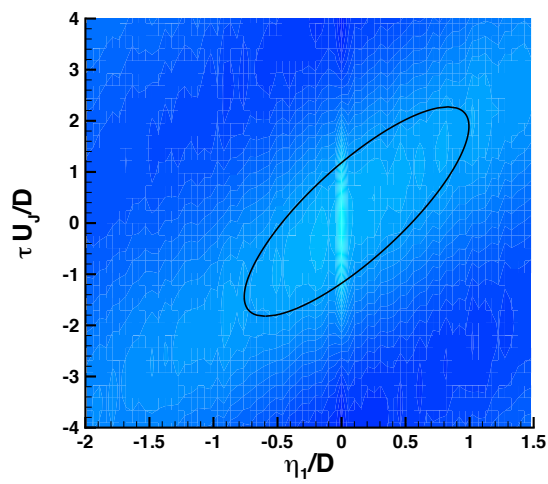

Estimate:

$$
\begin{aligned}
& L_{\eta}=\int_{0}^{+\infty} r_{11}\left(\boldsymbol{x}, \eta_{1}, \tau=0\right) \mathrm{d} \eta_{1} \\
& \tau_{\eta}=\int_{0}^{+\infty} r_{11}\left(\boldsymbol{x}, \eta_{1}=U_{c} \tau, \tau\right) \mathrm{d} \tau \\
& \text { using ellipse eq. }
\end{aligned}
$$

\begin{tabular}{l|lllll} 
& Total & IMF 1 & IMF 2 & IMF 3 & IMF 4 \\
$U_{c} / U_{J}$ & $\mathbf{0 . 5 9 6}$ & 0.670 & $\mathbf{0 . 5 8 6}$ & $\mathbf{0 . 5 4 0}$ & $\mathbf{0 . 3 8 4}$ \\
$L_{\eta} / D$ & $\mathbf{0 . 3 6 6}$ & 0.076 & $\mathbf{0 . 2 0 6}$ & $\mathbf{0 . 3 7 5}$ & $\mathbf{0 . 5 4 5}$ \\
$\tau_{\eta} U_{J} / D$ & $\mathbf{3 . 0 5 7}$ & 0.403 & $\mathbf{1 . 0 3 6}$ & $\mathbf{2 . 2 4 6}$ & $\mathbf{2 . 2 6 6}$
\end{tabular}

High frequency, small-scale for subgrid model 


\section{Frequency Dependent Values}

Compute complex coherence $\gamma_{i j}$ (Kerhervé et al. (2006))

Length scale $\Lambda_{11}^{1}(\boldsymbol{x}, \omega)=\int_{0}^{+\infty} \Re\left\{\gamma_{11}\left(\boldsymbol{x}, \eta_{1}, \omega\right)\right\} \mathrm{d} \eta_{1}$

Time scale $\tau_{11}^{1}(\boldsymbol{x}, \omega)=\frac{1}{u_{c 1}(\omega)} \int_{0}^{+\infty}\left|\gamma_{11}\left(\boldsymbol{x}, \eta_{1}, \omega\right)\right| \mathrm{d} \eta_{1}$

Phase velocity $\quad u_{c 1}(\omega)=\omega /\left|\partial \phi\left(\eta_{1}, \omega\right) / \partial \eta_{1}\right|$

- Scales difficult to compute without modeling, ex. $\Re\left\{\gamma_{11}\right\}$ oscillates, integration difficult

- Estimate length scale from $\left|\gamma_{11}\right|$ decays by $1 / e$

- Estimate time scale from $\left|\gamma_{11}\right| / u_{c 1}$ decays by $1 / \mathrm{e}$ 


\section{Frequency Dependent Phase Velocity}

Issues: Higher IMFs (lower frequency) lack resolution, low number of averages

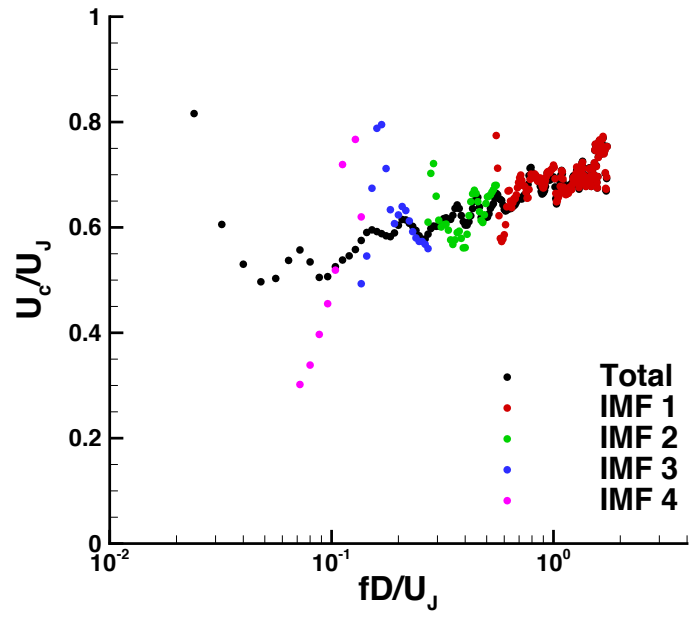

Ref. Pt.:

$\mathrm{x} / \mathrm{D}=7.82$

$\mathrm{r} / \mathrm{D}=-0.49$

end of potential core 


\section{Frequency Dependent Phase Velocity}

Compared to ellipse eq. based phase velocity

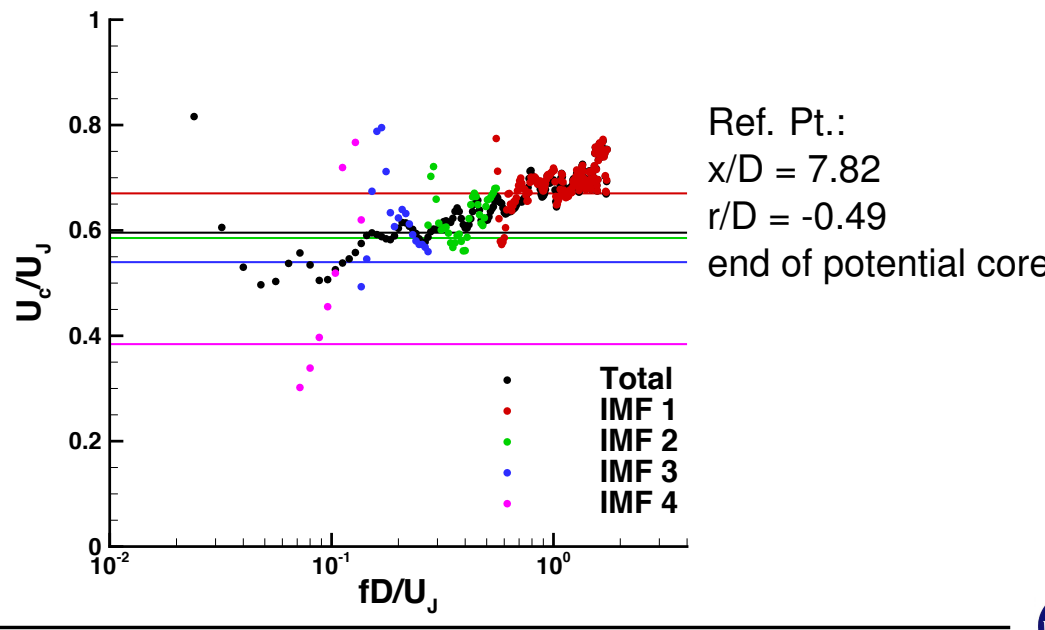




\section{Frequency Dependent Phase Velocity}

Compared to ellipse eq. based phase velocity

Compared to Morris \& Zaman (2010) fit, $\mathrm{M}_{J}=0.25$ jet

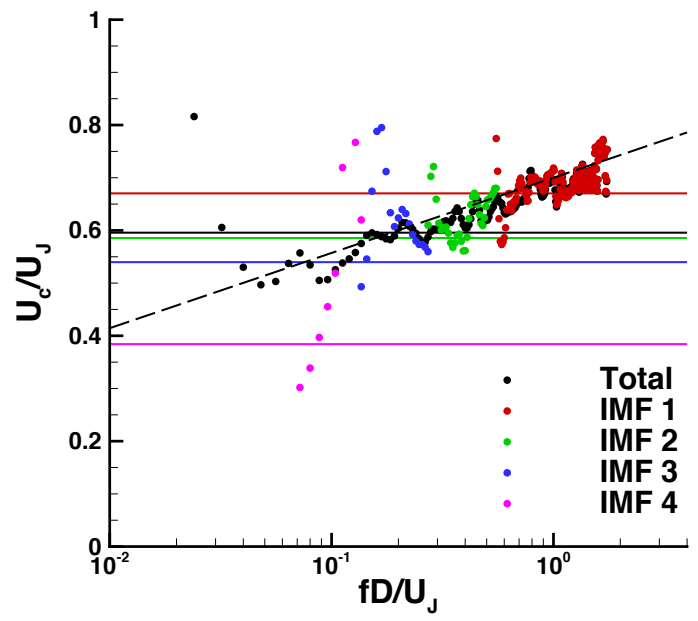

Ref. Pt.:

$\mathrm{x} / \mathrm{D}=7.82$

$r / D=-0.49$

end of potential core 


\section{Frequency Dependent Phase Velocity}

Compared to ellipse eq. based phase velocity

Compared to Morris \& Zaman (2010) fit, $\mathrm{M}_{J}=0.25$ jet

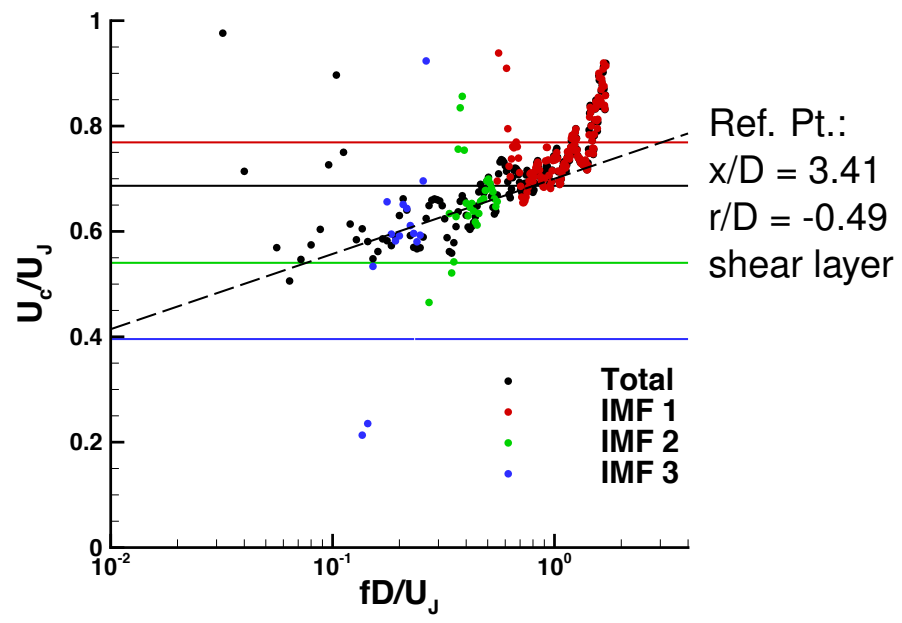




\section{Frequency Dependent Phase Velocity}

Compared to ellipse eq. based phase velocity

Compared to Morris \& Zaman (2010) fit, $\mathrm{M}_{J}=0.25$ jet

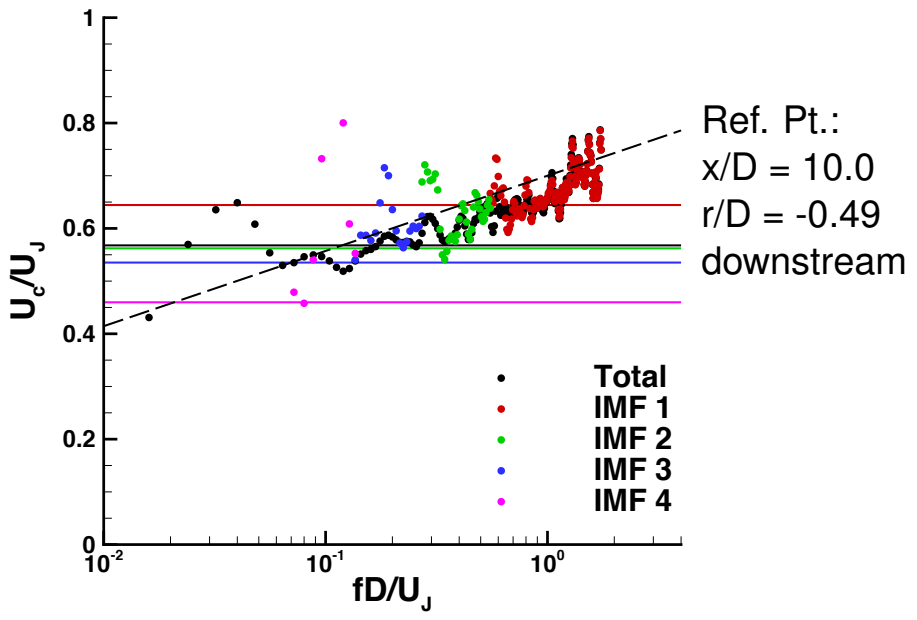




\section{Frequency Dependent Length Scale}

- Compared to ellipse eq. based length scale

- Using $\left|\gamma_{11}\right|$ instead of $\Re\left\{\gamma_{11}\right\}$ over-estimates the length scale

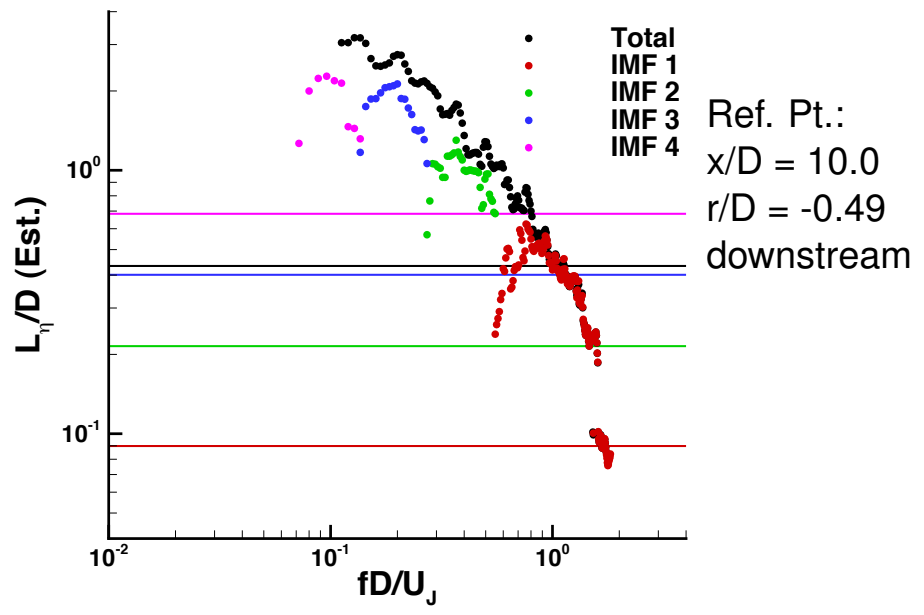




\section{Frequency Dependent Time Scale}

- Compared to ellipse eq. based time scale (solid)

- Direct interpolated value from correlation function (dashed)

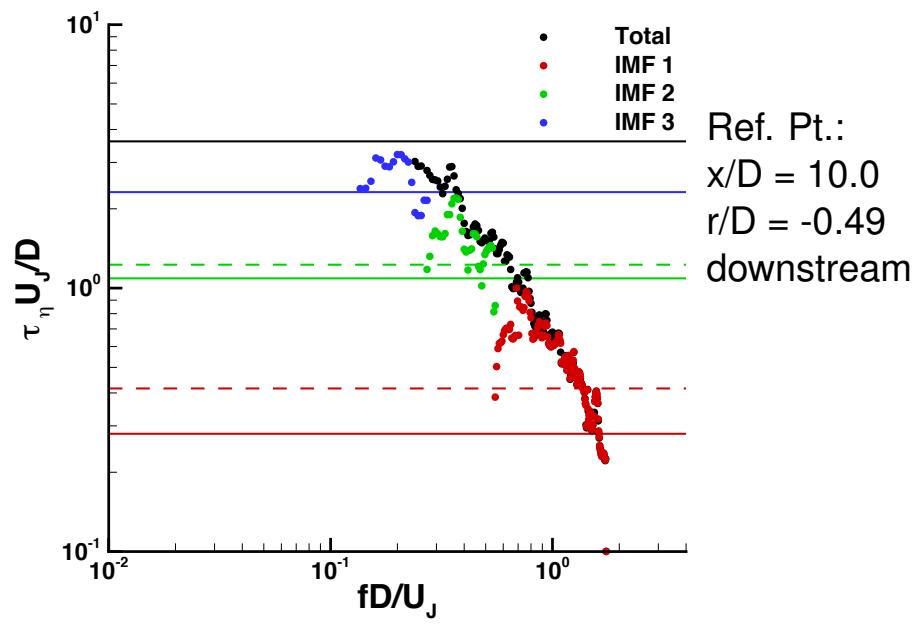




\section{IMF Orthogonality and Correlation}

Accepted that IMFs nearly orthogonal and uncorrelated at a point

$$
r_{11}\left(\boldsymbol{x}, \eta_{1}, \tau\right)=\frac{\overline{u_{1}^{\prime}(\boldsymbol{x}, t) \cdot u_{1}^{\prime}\left(\boldsymbol{x}+\eta_{1}, t+\tau\right)}}{\sqrt{\overline{u_{1}^{\prime}(\boldsymbol{x}, t)^{2}} \cdot \overline{u_{1}^{\prime}\left(\boldsymbol{x}+\eta_{1}, t+\tau\right)^{2}}}}=\sum_{n=1}^{N} \frac{\overline{C_{1 n}(\boldsymbol{x}, t) \cdot C_{1 n}\left(\boldsymbol{x}+\eta_{1}, t+\tau\right)}}{\sqrt{\overline{u_{1}^{\prime}(\boldsymbol{x}, t)^{2}} \cdot \overline{u_{1}^{\prime}\left(\boldsymbol{x}+\eta_{1}, t+\tau\right)^{2}}}}
$$

Separate

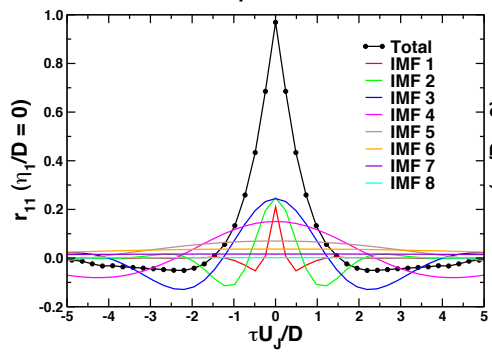

Summed

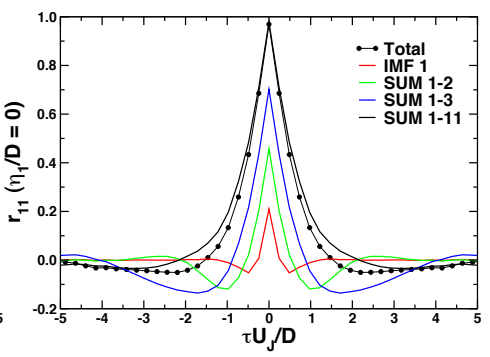




\section{IMF Orthogonality and Correlation}

What about the correlation between two points?

$$
\begin{aligned}
r_{11}\left(\boldsymbol{x}, \eta_{1}, \tau\right)=\frac{\overline{u_{1}^{\prime}(\boldsymbol{x}, t) \cdot u_{1}^{\prime}\left(\boldsymbol{x}+\eta_{1}, t+\tau\right)}}{\sqrt{\overline{u_{1}^{\prime}(\boldsymbol{x}, t)^{2}} \cdot \overline{u_{1}^{\prime}\left(\boldsymbol{x}+\eta_{1}, t+\tau\right)^{2}}}} & =\sum_{n=1}^{N} \frac{\overline{C_{1 n}(\boldsymbol{x}, t) \cdot C_{1 n}\left(\boldsymbol{x}+\eta_{1}, t+\tau\right)}}{\sqrt{\overline{u_{1}^{\prime}(\boldsymbol{x}, t)^{2}} \cdot \overline{u_{1}^{\prime}\left(\boldsymbol{x}+\eta_{1}, t+\tau\right)^{2}}}} \\
& + \text { cross-correlation terms }
\end{aligned}
$$

Separate

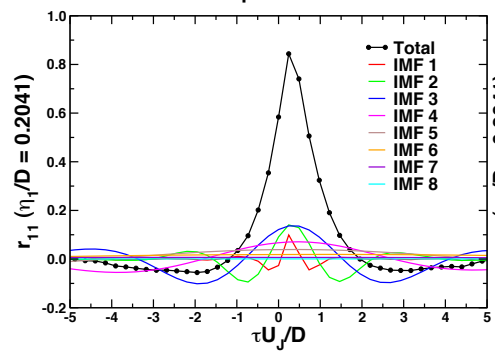

Summed

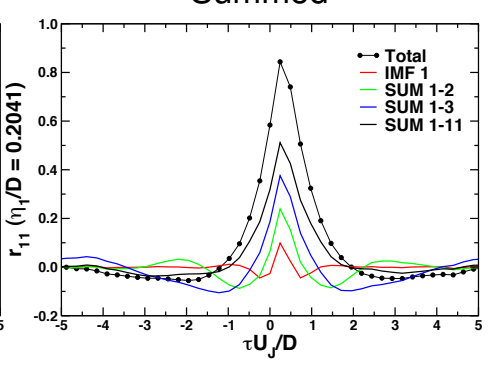




\section{Concluding Remarks}

- New application of empirical mode decomposition

- equivalent to passing array of time histories through a bank of band-pass filters - broadband signals only

- highest frequency range turbulent statistics may be applicable to subgrid modeling

- Issues:

- higher sampling rates: higher Strouhal numbers, finer time resolution at high frequencies

- longer time histories: greater frequency resolution, increased number of averages

- finer spatial resolution: capture high frequency changes in a short distance

- Further analysis:

- IMF cross-correlation terms

- Other approaches to using EMD results 
